\title{
Spectral Element Method for Damage Localization in Non-Uniform Structures with Parametric Uncertainty
}

\author{
Purushottam Kumar Chaudhary $^{1}$, Kumar Anjneya ${ }^{2}$, Koushik Roy ${ }^{3}$ \\ ${ }^{1,3}$ Department of Civil and Environmental Engineering, IIT Patna \\ India-801103 \\ First.pkchy31.pc@yahoo.com; Third.koushik@iitp.ac.in \\ ${ }^{2}$ Department of Civil Engineering, IIT Kharagpur \\ India, 721302 \\ Second.Kanjneya@gmail.com
}

\begin{abstract}
Spectral element method, which works in the frequency domain, is usually used for modelling structures. It is a wave-based numerical approach. The advantage of using this method is that it reduces the number of degrees-of-freedom by representing the whole structure using a single element. The present study deals with finding damage in non-uniform structures using the spectral element method. Since the uncertainty is part and parcel of every real structure, it needs to be introduced into every methodology meant for damage detection. Therefore, this method is also used here for the stochastic system to introduce uncertainty in the model. Damage at any location is introduced as a single edge notch crack. The crack introduced is a non-propagating crack. The damage quantity introduced is expressed in terms of crack flexibility-based on the concept of fracture mechanics. At the damage location, the compatibility conditions are to be satisfied. With the help of the boundary as well as compatibility conditions being satisfied, the displacement equation for damaged case in the frequency domain is developed. In order to introduce parametric uncertainty, expressions of spectral stiffness and mass matrices are established using Karhunen-Loéve expansion. The mass and stiffness matrices for the stochastic case are expressed as random field and are discretized in terms of the random variables. Eigenvalue analysis is performed to obtain mode shapes for both damaged and undamaged cases. Difference between the mode shapes is considered to obtain the damage location. The methodology is found to be effective in localizing damage in non-uniform structures in the presence of parametric uncertainty.
\end{abstract}

Keywords: Spectral element method, Karhunen-Loéve (KL) expansion, Damage localization, ' Non-uniform structure, Parametric uncertainty, Random field

\section{Introduction}

SHM is a process of observing a structure over the course of time. These studies are used to determine the present state of the structure. In the case of damage, the modal properties (i.e. frequencies and mode shapes) change and these are compared with that of its undamaged state values to localize and quantify the damage. In the process of inverse optimization, the inverse algorithm is used to obtain the model parameters like stiffness. The measurements taken from the actual structures are the displacement or the acceleration responses, which are used to extract the mode shapes and the frequencies through fast Fourier transformation (FFT) or frequency domain decomposition (FDD). These are then used to obtain the stiffness or other model properties. There are lots of work done in this field using various techniques, and different level of success has been achieved in these fields [1]. The spectral element method (SEM) has added advantages over these techniques. The concept of SEM was given by Beskos [2] in 1978, which includes the key benefits of the Finite element method (FEM), Dynamic stiffness method (DSM) and Spectral analysis method (SAM). In the SEM, [3] the stiffness matrix is established in the frequency domain. The whole structural model is developed as a single element in the frequency domain, thus reducing the degrees of freedom. Eigenvalues and eigenvectors are the exact solutions. Also, SEM lowers the computational time and cost as it requires fewer degrees of freedom. SEM [3] is based on the analytical solution of the displacement wave equation in the frequency domain. In this method, the interpolation function is the exact solution of the wave equation. There are, however, some of the drawbacks associated with this method. Complex geometries cannot be modelled using this method as wave Eqs. are not available for the complex geometries. This method again cannot be used for the nonlinear system because the superposition principle is not applicable to the nonlinear systems. In any of the damage identification procedures, 
uncertainties are inherently involved in the parameters, modelling, and measurements. All these kind of uncertainty limits the use of deterministic damage detection techniques. If the level of uncertainty is high, then the actual information regarding the damage condition may get concealed, and the damage may not be identified. In other words, this may lead to negative falsity, where actual damage is not detected, or positive falsity, where false damage is detected, in the intact structural element. When uncertainty is considered as the random variable, then Monte Carlo simulation [4] is used. While if it is used as the random process, then Karhunen- Loéve (KL) expansion decomposition needs to be applied. Monte Carlo simulation is a sampling method which generates the independent random variables based on their distribution. For each of these values, the deterministic problem is solved to obtain the solution. Considering random field, Karhunen Loéve (KL) expansion is used to discretize the random field by representing it using the random variables and continuous deterministic functions. In work by Machado et al. [5], they developed the spectral element method for the rod element free at both the ends. They also studied the damage detection capability of SEM under the stochastic condition. They used KL expansion to introduce uncertainty into the system. In work by Adhikari and Friswell [6], KL expansion was used for distributed parameter model updating. In this work, the parameters to be updated were expressed as the spatially correlated random fields. Ostachowicz [7] presented the overall process of equation generation, damage modelling and elastic wave propagation using SEM in a review form. In the present study, the spectral element method is used to derive the governing frequencies, and mode shapes Eqs. in the frequency domain for a non-uniform structure. This is used for damage detection in stochastic cases. In the present study, uncertainty has been introduced into the structural parameters by expressing them as spatially uncorrelated random variables. The random fields corresponding to mass and stiffness matrices have been expanded using KL expansion. In this paper, first of all, the spectral element method has been used to develop the modal Eqs. in the frequency domain for a nonuniform section. After this, a study on stochastic spectral analysis of damaged tapered rectangular cross-section rod is presented. At the end of this paper, damage detection capability of the SEM under stochastic situation have been explored.

\section{Spectral Element Method for Non-Uniform Section}

Let there be both ends free tapered rectangular cross-section rod of length $\mathrm{L}$ and width $b$. Let the cross-sectional areas be $A_{1}$ and $A_{2}$ at node 1 and node 2, respectively, as shown in Fig. (1). Axial force $F_{1}$ and $F_{2}$ are acting in axial direction at node 1 and node 2 respectively. Again, $u_{1}$ and $u_{2}$ are the displacements at node 1 and node 2 .

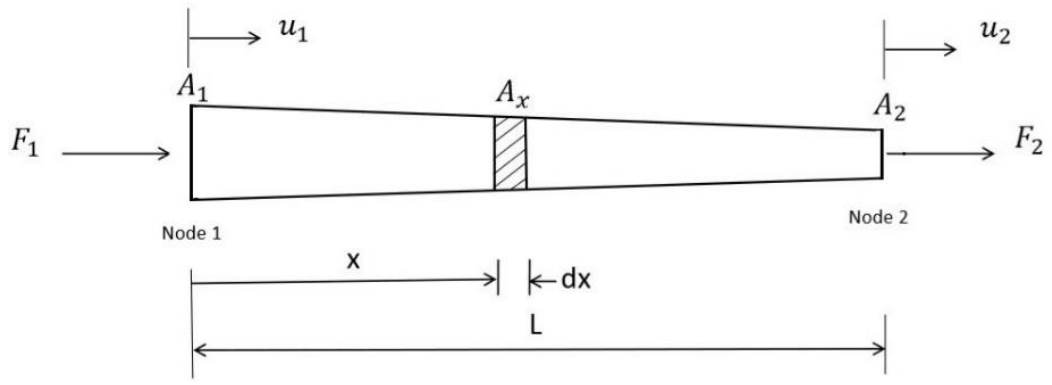

Fig.1: Tapered rectangular cross-section rod with both ends free.

Now, taking an elementary strip of length $d x$ at a distance $x$ from node 1 , the area of this elementary strip is given by the following equation.

$$
A_{x}=A_{1}+\frac{\left(A_{2}-A_{1}\right) x}{L}
$$

\subsection{Spectral Analysis of Undamaged Vibrating Rod}

The undamped equilibrium equation in the frequency domain is given by:

$$
E A \frac{d^{2} u(x)}{d x^{2}}+\omega^{2} \rho A u(x)=q(x)
$$

where, $A=$ cross-section area, $\rho=$ density, $E=$ modulus of elasticity, $u=$ longitudinal displacement, $q=$ distributed load and $\omega=$ circular frequency. 
Now, the solution as per Machado et al. [5] of equation (2) is given by,

$$
u(x)=a_{1} e^{-\imath k x}+a_{2} e^{-\imath k(L-x)}=s(x, \omega) a
$$

where, $\mathrm{s}(\mathrm{x}, \omega)=\left\{\mathrm{e}^{-\mathrm{ikx}} \mathrm{e}^{-\mathrm{ik}(\mathrm{L}-\mathrm{x})}\right\}, \mathrm{a}=\left\{\begin{array}{l}a_{1} \\ a_{2}\end{array}\right\}, a_{1}$ and $a_{2}$ are constants, $\mathrm{k}=\omega / \mathrm{c}=$ wavenumber corresponding to the longitudinal wave propagation, and $\mathrm{c}=\sqrt{\mathrm{E} / \rho}=$ phase speed. Now, the spectral nodal displacements of the rod are given by:

$$
d=\left\{\begin{array}{l}
u_{1} \\
u_{2}
\end{array}\right\}=\left\{\begin{array}{l}
u(0) \\
u(L)
\end{array}\right\}=\left[\begin{array}{l}
s(0, \omega) \\
s(L, \omega)
\end{array}\right] a=G(\omega) a
$$

Here,

$$
G(\omega)=\left[\begin{array}{cc}
1 & e^{-\imath k L} \\
e^{-\imath k L} & 1
\end{array}\right]
$$

Substituting equation (4) into Equation (3) the deformation at any point in the rod is obtained as follows:

$$
u(x, \omega)=s(x, \omega) G^{-1}(\omega) d=\left\{\begin{array}{ll}
g_{1} & g_{2}
\end{array}\right\} d
$$

Consider an undamaged tapered rectangular cross-section rod with one end fixed and another end free, as shown in Fig. (2). Rod with one end free and other end fixed will have $u_{1}$ equal to zero while $u_{2}$ will have nonzero value and hence the corresponding reduced dimension of $G^{-1}(\omega)$ will be obtained by eliminating its first column, i.e. $G^{-1}(\omega)$ will have a dimension of $2 \times 1$ and it is denoted by $G_{n}{ }^{-1}(\omega)$.

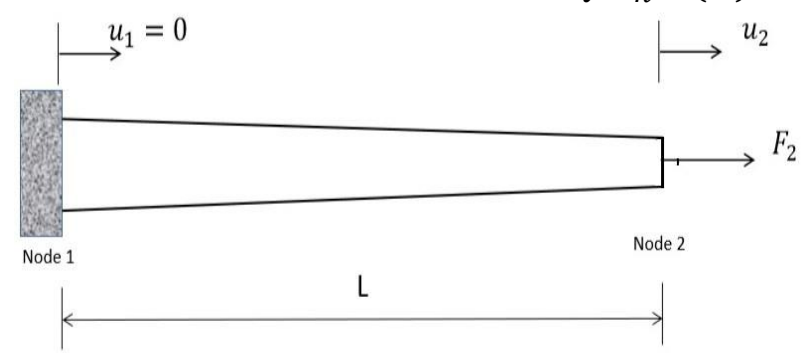

(i)

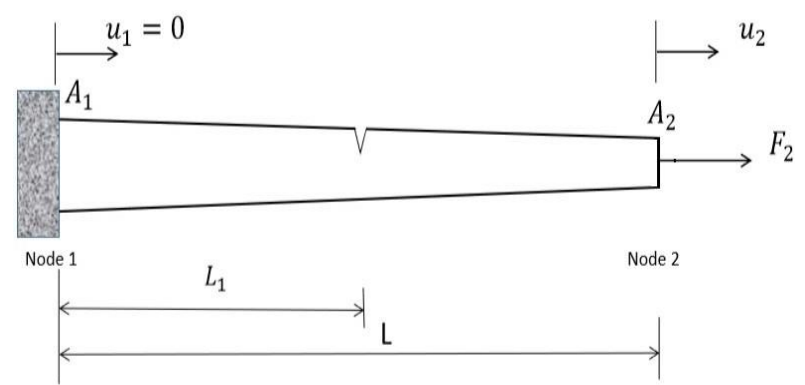

(ii)

Fig. 2: (i) Undamaged tapered rectangular cross-section rod with one end fixed and another end free, (ii) Damaged tapered rectangular cross-section rod with one end fixed and another end free

The displacement in generalized form is given by $u(x)=g_{2}(x) u_{2}=\frac{\sin (k x)}{\sin (k L)} u_{2}$. The stiffness matrix and mass matrix are given by following Eqs.

$$
\begin{aligned}
K_{N u}(\omega) & =E \Gamma_{n}^{T}(\omega)\left[\int_{0}^{L} A_{x} s^{T}(x, \omega) s^{\prime}(x, \omega) d x\right] \Gamma_{n}(\omega) \\
& =K_{N u_{11}} \\
M_{N u}(\omega) & =\rho \Gamma_{n}^{T}(\omega)\left[\int_{0}^{L} A_{x} s^{T}(x, \omega) s(x, \omega) d x\right] \Gamma_{n}(\omega) \\
& =M_{N u_{11}}
\end{aligned}
$$

Where, $(\bullet)^{\prime}$ represents the first derivative w.r.t x, $\Gamma_{n}(\omega)=G_{n}{ }^{-1}(\omega), s(x, \omega)$ is similar to one given earlier, while $K_{N u_{11}}$ and $M_{N u_{11}}$ can be referred to Machado et al.[4].

\subsection{Spectral Analysis of Damaged Tapered Rod}

Consider a damaged tapered rectangular cross-section rod with one end fixed and another end free, as shown in Fig. (2). Also, assume that there is a single edge notch crack of depth $a$ at length $L_{1}$ from node 1 , as shown in Fig. (2). The damaged 
rod is formulated as a function of crack flexibility $(\Theta)$ by using Castigliano's theorem and the laws of fracture mechanics. Now, for damaged rod, the solution of equation (2) is given in two parts. One part is, for the length between fixed end to crack position, i.e. left part of the crack and another part is between a crack position to free end.

The solution by Machado et al. [5], is given by following Eqs.

$$
\begin{aligned}
u_{L}(x) & =a_{1} e^{-l k x}+a_{2} e^{-l k\left(L_{1}-x\right)} \quad\left(0 \leq x \leq L_{1}\right) \\
& =s_{L}(x, \omega) a_{L}
\end{aligned}
$$

where,

$$
\begin{aligned}
& s_{L}(x, \omega)=\left[\mathrm{e}^{-\mathrm{ikx}} \mathrm{e}^{-\mathrm{ik}(\mathrm{L} 1-\mathrm{x})}\right], \text { and } a_{L}=\left\{\begin{array}{l}
a_{1} \\
a_{2}
\end{array}\right\} \\
u_{R}(x) & =a_{3} e^{-\imath k x}+a_{4} e^{-\imath k(L-x)} \quad\left(L_{1} \leq x \leq L\right) \\
& =s_{R}(x, \omega) a_{R}
\end{aligned}
$$

where $s_{R}(x, \omega)=\left[\mathrm{e}^{-\mathrm{ikx}} \mathrm{e}^{-\mathrm{ik}(\mathrm{L}-\mathrm{x})}\right]$, and $a_{R}=\left\{\begin{array}{l}a_{3} \\ a_{4}\end{array}\right\}$. Now, the Eqs. (9a-9c) in the combined form are given by the following equation.

$$
\left\{\begin{array}{l}
u_{L}(x) \\
u_{R}(x)
\end{array}\right\}=\left[\begin{array}{cc}
s_{L}(x, \omega) & 0 \\
0 & s_{R}(x, \omega)
\end{array}\right]\left\{\begin{array}{l}
a_{L} \\
a_{R}
\end{array}\right\}=s_{d}(x, \omega) a_{d}
$$

Here, $a_{d}$ is calculated by using the following boundary and compatibility conditions [5]:
a) $u_{L}(0)=u_{1}$;
b) $u_{R}\left(L_{1}\right)-u_{L}\left(L_{1}\right)=\Theta \frac{\partial u_{L}\left(L_{1}\right)}{\partial x}$;
c) $\frac{\partial u_{L}\left(L_{1}\right)}{\partial x}=\frac{\partial u_{R}\left(L_{1}\right)}{\partial x}$
d) $u_{R}(L)=u_{2}$.

Now, from Eqs. (9a-9c) and boundary conditions the following Eqs. can be obtained.

$$
\begin{aligned}
& {\left[\begin{array}{cccc}
1 & e^{-\imath k L_{1}} & 0 & 0 \\
(\iota k \Theta-1) e^{-\imath k L_{1}} & -(\iota k \Theta+1) & e^{-\imath k L_{1}} & e^{-\imath k\left(L-L_{1}\right)} \\
-\iota k e^{-\imath k L_{1}} & \iota k & \iota k e^{-\imath k L_{1}} & -\iota k e^{-\imath k\left(L-L_{1}\right)} \\
0 & 0 & e^{-\imath k L} & 1
\end{array}\right]\left\{\begin{array}{l}
a_{1} \\
a_{2} \\
a_{3} \\
a_{4}
\end{array}\right\}=\left\{\begin{array}{c}
u_{1} \\
0 \\
0 \\
u_{2}
\end{array}\right\}} \\
& \Rightarrow G_{d} a_{d}=u_{d} \Rightarrow a_{d}=G_{d}{ }^{-1} u_{d}
\end{aligned}
$$

Rod with both ends free condition will have nonzero $u_{1}$ and $u_{2}$ and hence the corresponding reduced dimension of $G_{d}{ }^{-1}$ will be obtained by eliminating its second and third column, i.e. $G_{d}{ }^{-1}$ will have a dimension of $4 \times 2$ and it is denoted by $G_{d_{1}}{ }^{-1}$. By substituting Equation (12) into equation (10),

$$
\left\{\begin{array}{l}
u_{L}(x) \\
u_{R}(x)
\end{array}\right\}=\left[\begin{array}{cc}
s_{L}(x, \omega) & 0 \\
0 & s_{R}(x, \omega)
\end{array}\right] G_{d 1}{ }^{-1} u_{d}=\left[\begin{array}{ll}
g_{11} & g_{12} \\
g_{21} & g_{22}
\end{array}\right]\left\{\begin{array}{l}
u_{1} \\
u_{2}
\end{array}\right\}
$$

where, $g_{11}, g_{12}, g_{21}$ and $g_{22}$ are given by following Eqs.

$$
\begin{gathered}
g_{11}=\frac{\begin{array}{c}
\cos (k L 1)+i \sin (k L 1))(-i \cos (k L-k L 1)+\Theta k \cos (k L-k L 1)+ \\
\sin (k L 1)(\cos (k x)+\cos (k L 1-k x)-i \sin (k x)-i \sin (k L 1-k x)
\end{array}}{\Theta k \cos (k L)+\Theta k \cos (k L-2 k L 1)+2 \sin (k L)} \\
g_{12}=\frac{2 \sin (k x)}{\Theta k \cos (k L)+\Theta k \cos \left(k L-2 k L_{1}\right)+2 \sin (k L)}
\end{gathered}
$$




$$
\begin{gathered}
g_{21}=\frac{\begin{array}{c}
\cos (k L 1)+i \sin (k L 1))(-i \cos (k L-k L 1)+\Theta k \cos (k L-k L 1)+ \\
\sin (k L-k L 1)(\cos (k x)+\cos (k L 1-k x)-i \sin (k x)-i \sin (k L-k x)
\end{array}}{\Theta k \cos (k L)+\Theta k \cos (k L-2 k L 1)+2 \sin (k L)} \\
g_{22}=\frac{\Theta k \cos (k x)+\Theta k \cos \left(2 k L_{1}-k x\right)+2 \sin (k x)}{\Theta k \cos (k L)+\Theta k \cos \left(k L-2 k L_{1}\right)+2 \sin (k L)}
\end{gathered}
$$

For a rod with one end fixed and another end free $u_{1}$ is equal to zero, while $u_{2}$ will have nonzero value and hence the corresponding reduced dimension of $G_{d}{ }^{-1}(\omega)$ will be obtained by eliminating its first, second and third column, i.e. $G_{d}{ }^{-1}(\omega)$ will have a dimension of $4 \times 1$ and it is denoted by $G_{d_{2}}{ }^{-1}(\omega)$.

The stiffness matrix and mass matrix are given by following Eqs.

$$
\begin{aligned}
K_{N d}(\omega) & =E \Gamma_{d}^{T}(\omega)\left[\begin{array}{cc}
\int_{0}^{L_{1}} A_{x} s_{L}^{\prime T}(x, \omega) s_{L}^{\prime}(x, \omega) d x & 0 \\
0 & \int_{L_{1}}^{L} A_{x} s_{R}^{\prime T}(x, \omega) s_{R}^{\prime}(x, \omega) d x
\end{array}\right] \Gamma_{d}(\omega) \\
& =\left[K_{\left.N d_{11}\right]}\right. \\
M_{N d}(\omega) & =\rho \Gamma_{d}^{T}(\omega)\left[\begin{array}{cc}
\int_{0}^{L_{1}} A_{x} s_{L}^{T}(x, \omega) s_{L}(x, \omega) d x & 0 \\
0 & \int_{L_{1}}^{L} A_{x} s_{R}^{T}(x, \omega) s_{R}(x, \omega) d x
\end{array}\right] \Gamma_{d}(\omega)
\end{aligned}
$$

where $\Gamma_{d}(\omega)=G_{d 2}{ }^{-1}$, and $K_{N d_{11}}$ and $M_{N d_{11}}$ can be referred from Machado et al. [5].

\section{Stochastic Spectral Element Approach for Non-Uniform Section} 3.1. Stochastic Spectral Element Analysis of Undamaged Tapered Rod with One End Fixed and another End Free.

To obtain the stiffness and mass matrices associated with the random components, for each $\boldsymbol{j}$ (i.e. uncorrelated random variable), matrices are obtained as follows [8].

$$
K(\omega, \theta)=K_{N u}(\omega)+\Delta K(\omega, \theta) ; \quad M(\omega, \theta)=M_{N u}(\omega)+\Delta M(\omega, \theta)
$$

where $K_{N u}(\omega)$ and $M_{N u}(\omega)$ are the deterministic part. Also, $\Delta K(\omega, \theta)$ and $\Delta M(\omega, \theta)$ are the random part of the stiffness and mass matrices respectively which is given by using KL expansion and are expressed as,

$$
\begin{gathered}
\Delta K(\omega, \theta)=\epsilon_{1} \sum_{j=1}^{N} \xi_{K j}(\theta) \sqrt{\lambda_{K j}} K_{j}(\omega) \\
\Delta M(\omega, \theta)=\epsilon_{2} \sum_{j=1}^{N} \xi_{M j}(\theta) \sqrt{\lambda_{M j}} M_{j}(\omega)
\end{gathered}
$$


where $\mathrm{N}$ is the number of terms kept after truncation of the infinite series in the KL expansion, while $\xi_{K j}(\theta)$ and $\xi_{M j}(\theta)$ are uncorrelated Gaussian random variables with zero mean and unit standard deviation.

The matrices $K_{j}(\omega)$ and $M_{j}(\omega)$ are written as

$$
\begin{aligned}
& K_{j}(\omega)=E \Gamma^{T}(\omega)\left[\int_{0}^{L} f_{K j}(x) A_{x} s^{\prime T}(x, \omega) s^{\prime}(x, \omega) d x\right] \Gamma(\omega) \\
& M_{j}(\omega)=\rho \Gamma^{T}(\omega)\left[\int_{0}^{L} f_{M j}(x) A_{x} s^{T}(x, \omega) s(x, \omega) d x\right] \Gamma(\omega)
\end{aligned}
$$

where, $s(x, \omega)$ is similar to the deterministic part. The random element of the stiffness and mass matrices are obtained by substituting the following values

and

$$
\left\{f_{j}\right\}(x)=\frac{\left\{\cos \left(\left\{\omega_{k}\right\}_{j} x\right)\right\}}{\sqrt{\left\{a+\frac{\left\{\sin \left(2\left\{\omega_{k}\right\}_{j} a\right)\right\}}{\left\{2\left\{\omega_{k}\right\}_{j}\right\}}\right\}}} \quad j=1,3,5, \ldots . .
$$

in Eqs. (23) and (24).

$$
\left\{f_{j}\right\}(x)=\frac{\left\{\sin \left(\left\{\omega_{k}\right\}_{j} x\right)\right\}}{\sqrt{\left\{a-\frac{\left\{\sin \left(2\left\{\omega_{k}\right\}_{j} a\right)\right\}}{\left\{2\left\{\omega_{k}\right\}_{j}\right\}}\right\}}} \quad j=2,4,6, \ldots . .
$$

The stiffness and mass matrices for odd $j$ are given by

$$
\begin{aligned}
K_{j}^{\text {odd }}(\omega) & =\frac{E}{\sqrt{a+\frac{\sin \left(2 \omega_{k_{j}} a\right)}{2 \omega_{k_{j}}}}} \Gamma^{T}(\omega)\left[\int_{0}^{L} A_{x} \cos \left(\omega_{k_{j}} x\right) s^{\prime T}(x, \omega) s^{\prime}(x, \omega) d x\right] \Gamma(\omega) \\
& =\frac{E}{\sqrt{a+\frac{\sin \left(\omega_{k_{j}} a\right)}{2 \omega_{k_{j}}}}}\left[K_{\left.o_{11}\right]}\right. \\
M_{j}^{\text {odd }}(\omega) & =\frac{\rho}{\sqrt{a+\frac{\sin \left(2 \omega_{k_{j}} a\right)}{2 \omega_{k_{j}}}}} \Gamma^{T}(\omega)\left[\int_{0}^{L} A_{x} \cos \left(\omega_{k_{j}} x\right) s^{T}(x, \omega) s(x, \omega) d x\right] \Gamma(\omega) \\
& =\frac{\rho}{\sqrt{a+\frac{\sin \left(2 \omega_{k_{j}} a\right)}{2 \omega_{k_{j}}}}}\left[M_{\left.o_{11}\right]}\right.
\end{aligned}
$$

The stiffness and mass matrices for even $j$ are given by

$$
\begin{aligned}
K_{j}^{\text {even }}(\omega) & =\frac{E}{\sqrt{a-\frac{\sin \left(2 \omega_{k_{j}} a\right)}{2 \omega_{k_{j}}}}} \Gamma^{T}(\omega)\left[\int_{0}^{L} \sin \left(\omega_{k_{j}} x\right) A_{x} s^{\prime T}(x, \omega) s^{\prime}(x, \omega) d x\right] \Gamma(\omega) \\
& =\frac{E}{\sqrt{a-\frac{\sin \left(2 \omega_{k_{j}} a\right)}{2 \omega_{k_{j}}}}}\left[K_{e_{11}}\right]
\end{aligned}
$$




$$
\begin{aligned}
M_{j}^{\text {even }}(\omega) & =\frac{\rho}{\sqrt{a-\frac{\sin \left(2 \omega_{k_{j}} a\right)}{2 \omega_{k_{j}}}}} \Gamma^{T}(\omega)\left[\int_{0}^{L} \sin \left(\omega_{k_{j}} x\right) A_{x} s^{T}(x, \omega) s(x, \omega) d x\right] \Gamma(\omega) \\
& =\frac{\rho}{\sqrt{a-\frac{\sin \left(2 \omega_{k_{j}} a\right)}{2 \omega_{k_{j}}}}}\left[M_{e_{11}}\right]
\end{aligned}
$$

where, $\Gamma(\omega)$ is given in section 2.1. The expression of the elements, $K_{o_{11}}, M_{o_{11}}, K_{e_{11}}$ and $M_{e_{11}}$ used in the above four Eqs. (27) to (30) can be referred to Machado et al.[5]. The stochastic spectral undamaged stiffness and mass matrices, $K(\omega, \theta)$ and $M(\omega, \theta)$, are obtained by substituting Eqs. (27) to (30) into the Eqs. (21) and (22) and then in the Eqs. (20).

\subsection{Stochastic Spectral Element Analysis Of Damaged Tapered Rod}

The stiffness and mass matrices for a non-uniform damaged rod with one end fix and another end free corresponding to the two eigenfunctions defined in Eqs. (25) and (26), are given by the following equations [8]

$$
K_{d}(\omega, \theta)=K_{N d}(\omega)+\Delta K_{d}(\omega, \theta) ; M_{d}(\omega, \theta)=M_{N d}(\omega)+\Delta M_{d}(\omega, \theta)
$$

where $K_{N d}(\omega)$ and $M_{N d}(\omega)$ are similar to the deterministic part, given in Eqs. (18) and (19) respectively while $\Delta K_{d}(\omega, \theta)$ and $\Delta M_{d}(\omega, \theta)$ are the random part of the stiffness and mass matrices which is given by using Karhunen Loéve expansion and is expressed as

$$
\begin{aligned}
& \Delta K_{d}(\omega, \theta)=\epsilon_{1} \sum_{j=1}^{N} \xi_{K j}(\theta) \sqrt{\lambda_{K j}} K_{j} d(\omega) \\
& \Delta M_{d}(\omega, \theta)=\epsilon_{2} \sum_{j=1}^{N} \xi_{M j}(\theta) \sqrt{\lambda_{M j}} M_{j} d(\omega)
\end{aligned}
$$

where $\mathrm{N}$ is the number of terms kept after truncation of the infinite series in the Karhunen Loéve expansion while $\xi_{K j}(\theta)$ and $\xi_{M j}(\theta)$ are uncorrelated Gaussian random variables with zero mean and unit standard deviation. The equation of deformation for left and right parts of the damaged rod is different, and hence the different limits of integration need to be considered for both sides.

The matrices $K_{j_{d}}(\omega)$ and $M_{j_{d}}(\omega)$ are given by

$$
\begin{gathered}
K_{j_{d}}(\omega)=E \Gamma_{d}^{T}(\omega)\left[\begin{array}{cc}
S k_{L} & 0 \\
0 & S k_{R}
\end{array}\right] \Gamma_{d}(\omega) \\
M_{j_{d}}(\omega)=\rho \Gamma_{d}^{T}(\omega)\left[\begin{array}{cc}
S m_{L} & 0 \\
0 & S m_{R}
\end{array}\right] \Gamma_{d}(\omega)
\end{gathered}
$$

where, $\Gamma_{d}(\omega)$ is given in section 2.2 and the matrices $S k_{L}, S k_{R}, S m_{L}$ and $S m_{R}$ are given by following equation.

$$
\begin{aligned}
& S k_{L}=\int_{0}^{L_{1}} f_{K j}(x) A_{x}{S^{\prime}}_{L}^{T}(x, \omega) s^{\prime}{ }_{L}(x, \omega) d x \\
& S k_{R}=\int_{L_{1}}^{L} f_{K j}(x) A_{x} s^{\prime}{ }_{R}^{T}(x, \omega) s_{R}^{\prime}(x, \omega) d x
\end{aligned}
$$




$$
\begin{gathered}
S m_{L}=\int_{0}^{L_{1}} f_{M j}(x) A_{x} s_{L}^{T}(x, \omega) s_{L}(x, \omega) d x \\
S m_{R}=\int_{L_{1}}^{L} f_{M j}(x) A_{x} s^{\prime}{ }_{R}^{T}(x, \omega) s^{\prime}{ }_{R}(x, \omega) d x
\end{gathered}
$$

where, $s_{L}(x, \omega)$ and $s_{R}(x, \omega)$ are similar to the deterministic part which are given in Eqs. (9a) and (9c), respectively. The matrices $S k_{L}, S k_{R}, S m_{L}$ and $S m_{R}$ for odd and even $j$ are obtained by substituting Eqs. (25) and (26) in Eqs. (36) to (39).

For odd $j$

$$
\begin{aligned}
& S k_{L}^{o d d}(\omega)=\frac{E}{\sqrt{a+\frac{\sin \left(2 \omega_{k_{j}} a\right)}{2 \omega_{k_{j}}}}}\left[\begin{array}{ll}
S k_{L_{o_{11}}} & S k_{L_{o_{12}}} \\
S k_{L_{o_{21}}} & S k_{L_{o_{22}}}
\end{array}\right] \\
& S k_{R}{ }^{o d d}(\omega)=\frac{E}{\sqrt{a+\frac{\sin \left(2 \omega_{k_{j}} a\right)}{2 \omega_{k_{j}}}}}\left[\begin{array}{ll}
S k_{R_{o_{11}}} & S k_{R_{o_{12}}} \\
S k_{R_{o_{21}}} & S k_{R_{o_{22}}}
\end{array}\right] \\
& S m_{L}{ }^{o d d}(\omega)=\frac{\rho}{\sqrt{a+\frac{\sin \left(2 \omega_{k_{j}} a\right)}{2 \omega_{k_{j}}}}}\left[\begin{array}{ll}
S m_{L_{o_{11}}} & S m_{L_{o_{12}}} \\
S m_{L_{21}} & S m_{L_{o_{2} 2}}
\end{array}\right] \\
& S m_{R}{ }^{o d d}(\omega)=\frac{\rho}{\sqrt{a+\frac{\sin \left(2 \omega_{k_{j}} a\right)}{2 \omega_{k_{j}}}}}\left[\begin{array}{ll}
S m_{R_{o_{11}}} & S m_{R_{o_{12}}} \\
S m_{R_{o_{21}}} & S m_{R_{o_{22}}}
\end{array}\right]
\end{aligned}
$$

For even $j$

$$
\begin{aligned}
S k_{L}{ }^{\text {even }}(\omega) & =\frac{E}{\sqrt{a-\frac{\sin \left(2 \omega_{k_{j}} a\right)}{2 \omega_{k_{j}}}}}\left[\begin{array}{ll}
S k_{L_{e_{11}}} & S k_{L_{e_{12}}} \\
S k_{L_{e_{21}}} & S k_{L_{e_{22}}}
\end{array}\right] \\
S k_{R}{ }^{\text {even }}(\omega) & =\frac{E}{\sqrt{a-\frac{\sin \left(2 \omega_{k_{j}} a\right)}{2 \omega_{k_{j}}}}}\left[\begin{array}{ll}
S k_{R_{e_{11}}} & S k_{R_{e_{12}}} \\
S k_{R_{21}} & S k_{R_{e_{22}}}
\end{array}\right] \\
S m_{L}{ }^{\text {even }}(\omega) & =\frac{\operatorname{\rho }}{\sqrt{a-\frac{\sin \left(2 \omega_{k_{j}} a\right)}{2 \omega_{k_{j}}}}}\left[\begin{array}{ll}
S m_{L_{e_{11}}} & S m_{L_{e_{12}}} \\
S m_{L_{e_{21}}} & S m_{L_{e_{22}}}
\end{array}\right]
\end{aligned}
$$




$$
S m_{R}{ }^{\text {even }}(\omega)=\frac{\rho}{\sqrt{a-\frac{\sin \left(2 \omega_{k_{j}} a\right)}{2 \omega_{k_{j}}}}}\left[\begin{array}{ll}
S m_{R_{e}} & S m_{R_{e_{12}}} \\
S m_{R_{12}} & S m_{R_{e_{22}}}
\end{array}\right]
$$

where, $S k_{L_{o_{i j}}}, S m_{L_{o_{i j}}}, S k_{R_{o_{i j}}}, S m_{R_{o_{i j}}}, S k_{L_{e} i j}, S m_{L_{e_{i j}}}, S k_{R_{e_{i j}}}$ and $S m_{R_{e_{i j}}}$ of these eight matrices can be referred from Machado et al.[5].

Now, the matrices $K_{j_{d}}(\omega)$ and $M_{j_{d}}(\omega)$ for odd and even $j$ are obtained by substituting Eqs. (40) to (47) in Eqs. (34) and (35). The random part of the stiffness and mass matrices for different $j$ are obtained by substituting Eqs. (34) and (35) in Eqs. (32) and (33).

\section{Stochastic Crack Localization}

Material and geometrical properties used for calculation purpose are: $E=200 \mathrm{GPa}, \rho=7850 \mathrm{~kg} / \mathrm{m}^{3}, L=1 \mathrm{~m}, b=$ $0.01 \mathrm{~m}, A_{1}=0.0005 \mathrm{~m}^{2}$ and $A_{2}=0.0003 \mathrm{~m}^{2}$. For the stochastic case, the properties used are: $\epsilon_{1}=\epsilon_{2}=0.01, a=2 \mathrm{~m}$ and $N=50$. Also $\xi_{K_{j}}$ and $\xi_{M_{j}}$ has taken as a set of 50 random variables with zero mean and unit standard deviation. Undamaged mode shape for the first three modes is shown in Fig. (3).

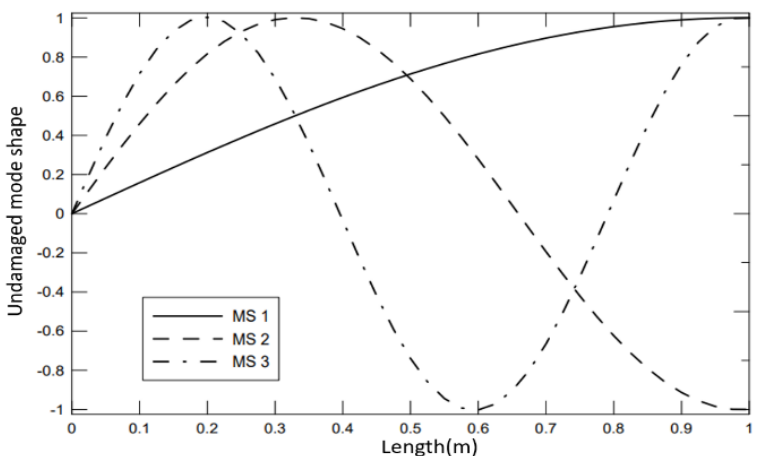

(i)

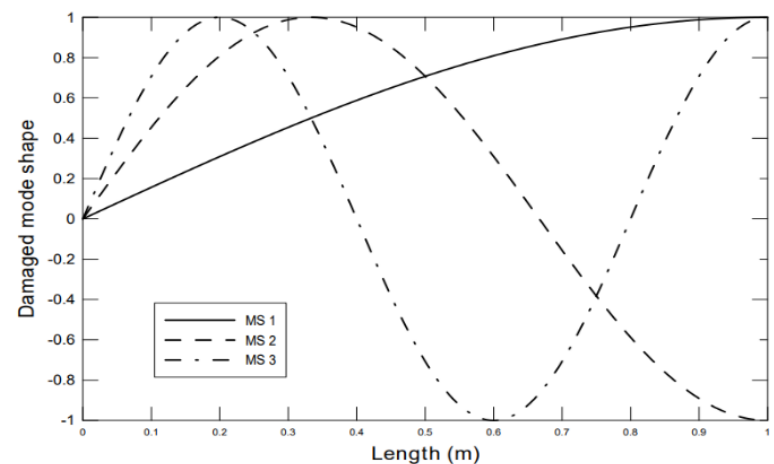

(ii)

Fig. 3: (i) Undamaged mode shape for the stochastic case, (ii) Damaged mode shape for stochastic the case for crack flexibility using stiffness degradation

Let us consider a damage at $0.7 \mathrm{~m}$ from fixed end with a stiffness degradation of $20 \%$. Corresponding to this condition, the damaged mode shape is shown in Fig. (3).

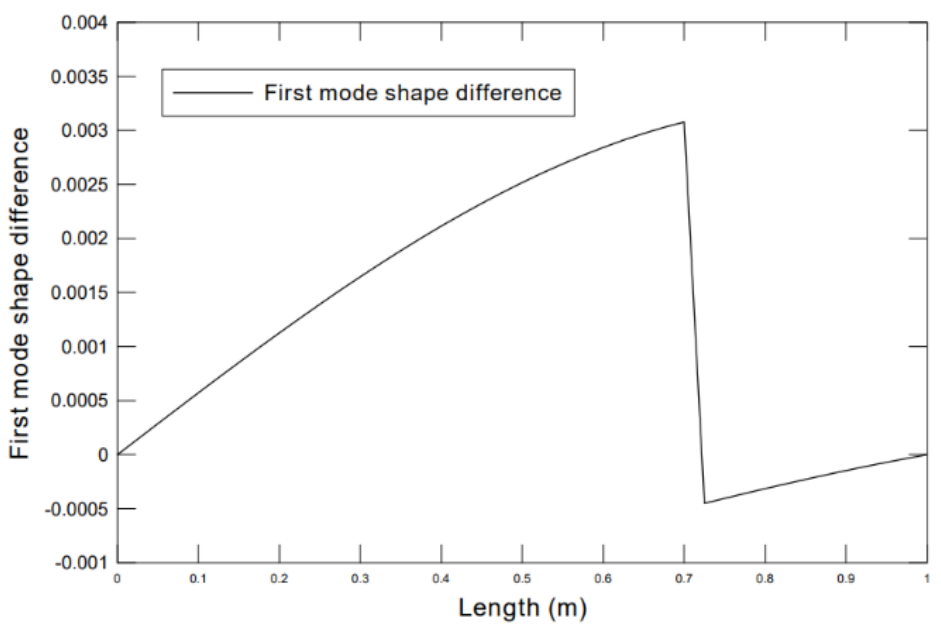

Fig. 4: Difference between first mode shape of damaged and undamaged state for $20 \%$ stiffness degradation 
Fig. 4 shows the difference between first mode shape of the damaged and undamaged state, [9] in which it can be seen clearly that it is abruptly changing at the assumed crack position, i.e. there is damage at $0.7 \mathrm{~m}$ from fixed end.

\section{Conclusion}

Spectral element method has been briefly explored in this paper for the non-uniform section. Stiffness and mass matrices for both damaged and undamaged case for the non-uniform section have been derived. The mass and the stiffness matrix have been expanded for the stochastic case using KL expansion decomposition. Eigenvalue analysis has been performed, and mode shapes for the damaged and undamaged stochastic case have been presented. Operating in the frequency domain the eigenvalues obtained are the exact solutions. Damaged localization has done after taking the difference between damaged and undamaged mode shape corresponding to the first mode. The first mode shape corresponding to the stochastic case have been plotted by taking an average of various uncertain mode shapes. The result shows that the method is successfully localizing the damage for the stochastic case. SEM helps to represent the whole structure as a single element. Further study is required to check its applicability in beams and frames.

\section{References}

[1] Y. L. Xu, J. Zhang, J. Li, and X. M. Wang, "Stochastic damage detection method for building structures with parametric uncertainties", Journal of sound and vibration, 330, pp. 4725-4737, 2011.

[2] G. V. Narayanan and D. E. Beskos, "Use of dynamic influence coefficients in forced vibration problem with the aid of fast Fourier transform", Computers and Structures, 9, pp. 145-150, 1978.

[3] M. Palacz, and M. Krawczuk, "Analysis of longitudinal wave propagation in a cracked rod by the spectral element method”, Computers and structures, 80, pp. 1809-1816, 2002.

[4] K. Binder, D. Heermann, Lyle Roelofs, A. John Mallinckrodt, and Susan McKay, "Monte Carlo Simulation in Statistical Physics", Computers in Physics, 7, 156, 1993.

[5] M. R. Machado, S. Adhikari and J. M. C. Santos, "A spectral approach for damage quantification in stochastic dynamic systems”, Mechanical systems and Signal Processing, 88, pp. 253-273, 2017.

[6] S. Adhikari and M. I. Friswell, "Distributed parameters model updating using the Karhunen-Loéve expansion", Mechanical systems and Signal Processing, 24, pp. 326-339, 2010.

[7] W. M. Ostachowicz, "Damage detection of structures using spectral finite element method", Computers and structures, 86, pp. 454-462, 2008.

[8] P. K. Chaudhary, K. Anjneya, D. Grover, and K. Roy, "Extraction of damage information in the presence of parametric uncertainty using dimensionality reduction", in Proceedings of the 29th European Safety and Reliability Conference, Hannover, Germany, 2019, pp. 3177-3182.

[9] K. Roy and S. Ray-Chaudhuri, "Fundamental mode shape and its derivatives in structural damage localization", Journal of sound and vibration, 332, pp. 5584-5593, 2013. 